\title{
Regulation of developmental rate and germ cell proliferation in Caenorhabditis elegans by the p53 gene network
}

\author{
WB Derry ${ }^{\star, 1,2,3}$, R Bierings ${ }^{1}$, M van lersel ${ }^{1}$, T Satkunendran ${ }^{2,3}$, V Reinke ${ }^{4}$ and JH Rothman ${ }^{\star, 1}$
}

Caenorhabditis elegans CEP-1 activates germline apoptosis in response to genotoxic stress, similar to its mammalian counterpart, tumor suppressor p53. In mammals, there are three p53 family members (p53, p63, and p73) that activate and repress many distinct and overlapping sets of genes, revealing a complex transcriptional regulatory network. Because CEP-1 is the sole p53 family member in C. elegans, analysis of this network is greatly simplified in this organism. We found that CEP-1 functions during normal development in the absence of stress to repress many (331) genes and activate only a few (28) genes. In response to genotoxic stress, 1394 genes are activated and 942 are repressed, many of which contain p53-binding sites. Comparison of the CEP-1 transcriptional network with transcriptional targets of the human p53 family reveals considerable overlap between CEP-1-regulated genes and homologues regulated by human p63 and p53, suggesting a composite p53/p63 action for CEP-1. We found that phg-1, the $C$. elegans Gas1 (growth arrest-specific 1) homologue, is activated by CEP-1 and is a negative regulator of cell proliferation in the germline in response to genotoxic stress. Further, we find that CEP-1 and PHG-1 mediate the decreased developmental rate and embryonic viability of mutations in the clk-2/TEL2 gene, which regulates lifespan and checkpoint responses.

Cell Death and Differentiation (2007) 14, 662-670. doi:10.1038/sj.cdd.4402075; published online 22 December 2006

The p53 tumor suppressor regulates a large number of genes that collaborate to modulate cell proliferation, DNA repair, apoptosis, and senescence; however, little is known about how this complex response is orchestrated to dictate various cellular outcomes in response to cellular stresses. ${ }^{1}$ Activation of p53 in response to DNA damage leads to cell cycle arrest or apoptosis, depending on the cell type and level of damage. ${ }^{1}$ p53 in mammals mediates arrest at G1, in part by activating the p21 ${ }^{\text {WAF1 }}$ cyclin-dependent kinase inhibitor. ${ }^{2}$ However, other mechanisms of p53-mediated cell cycle inhibition must exist, as p21-deficient fibroblasts are only partially defective in radiation-induced G1 arrest. ${ }^{3,4}$ Moreover, p53-mediated cell cycle arrest in Caenorhabditis elegans does not appear to involve the known p21 family members (our unpublished observations).

The presence of the p53 paralogs p63 and p73 in vertebrates complicates analysis of the p53 gene network. p63 and p73 can transactivate p53 target genes, ${ }^{5,6}$ and combined loss of both prevents cultured fibroblasts from undergoing genotoxicity-induced apoptosis, indicating that they act in p53-dependent processes. ${ }^{7}$ Although p63 and p73 are required for $\mathrm{p} 53$ to regulate some pro-apoptotic genes, recent findings challenge this apparent interplay between p53 family members, motivating studies of p53 function in a simpler system. ${ }^{8}$ A large number of p53-regulated genes that control cell cycle, apoptosis, cell adhesion, cytoskeletal function, metabolism, and intracellular signaling ${ }^{5,6,9-11}$ have been identified. Although there is considerable overlap in target genes regulated by all three p53 family members, there is significant variation among the genes identified in independent studies. ${ }^{9-12}$ These discrepancies are likely attributable to differences in tissue specificity, experimental conditions, and other parameters, thereby obscuring efforts to develop a coherent view of the $\mathrm{p} 53$ regulatory network.

C. elegans contains a single p53 family member, CEP-1 ( $C$. elegans p53-like-1), that regulates apoptosis and genome stability, ${ }^{13,14}$ making it an attractive system with which to study the p53 regulatory network. The CEP-1 DNA-binding domain is similar to that of human $p 53^{15}$ and CEP-1 binds to the p53 DNA consensus site. ${ }^{14,15}$ DNA damage in $C$. elegans is sensed by the HUS-1 and MRT-2 checkpoint proteins, which are required for genotoxicity-induced cell cycle arrest and CEP-1-dependent apoptosis of germ cells. ${ }^{16,17}$ CLK-2 acts in parallel with this checkpoint to transmit the damage signal to CEP-1 by an unknown mechanism. ${ }^{16,18}$ clk-2 mutants also show slow development and increased longevity, ${ }^{19,20}$ suggesting a regulatory role in developmental rate.

To illuminate CEP-1 function during normal development and in response to DNA damage, we analyzed transcriptional

\footnotetext{
${ }^{1}$ Department of Molecular, Cellular and Developmental Biology, University of California, Santa Barbara, CA, USA; ${ }^{2}$ Department of Medical Genetics and Microbiology, University of Toronto, Toronto, Ontario, Canada; ${ }^{3}$ Developmental and Stem Cell Biology Program, The Hospital for Sick Children, Toronto, Ontario, Canada and ${ }^{4}$ Department of Genetics, Yale University School of Medicine, New Haven, CT, USA

*Corresponding authors: WB Derry, The Hospital for Sick Children, Toronto Medical Discovery Tower, 101 College Street, Room 12-311, Toronto, Ontario, Canada M5G 1L7; E-mail: brent.derry@ sickkids.ca and JH Rothman, Department of Molecular, Cellular and Developmental Biology, University of California, Santa Barbara, CA 93106, USA. Tel: + 1805893 7885; Fax: + 1805893 2005; E-mail: rothman @lifesci.ucsb.edu

Keywords: p53; C. elegans; developmental timing; apoptosis; cell division; gene network

Abbreviations: Gro, slow growth; HU, hydroxy urea; IPTG, Isopropyl- $\beta$ - $D$-thiogalactopyranoside; NGM, nematode growth media; RNAi, RNA-mediated interference; UV, ultraviolet

Received 11.8.06; revised 30.10.06; accepted 30.10.06; Edited by V De Laurenzi; published online 22.12.06
} 
profiles of wild-type and cep-1 mutant animals. Many genes are regulated by CEP-1 in the absence of stress, revealing that CEP-1 functions during normal development and growth. We identified >1000 CEP-1-activated and 942 CEP-1repressed genes in response to UV radiation, and found conserved p53 binding sites in $\sim 25 \%$ of the upregulated genes. Comparison of our data with transcription profiles of p53, p63, and p73 in humans revealed substantial overlap between CEP-1- and p63-activated genes. In contrast, CEP-1 represses a number of genes whose homologues are repressed by mammalian p53. Both CEP-1 and the Gas1 homologue, PHG-1, which is activated by CEP-1 in response to UV radiation, are required for UV-induced arrest of germ cell proliferation, and both are required for the slower developmental progression and viability of clk-2 mutants. Thus, $C$. elegans p53 directs a wide range of cellular processes, including regulation of developmental rate in somatic cells.

\section{Results}

C. elegans p53 represses a large number of genes in the absence of stress. Although p53 is known to regulate many genes in response to various stresses, it is generally believed to be inactive in animals under normal growth conditions. We sought to determine whether CEP-1 regulates gene activity under nonstressed conditions by comparing transcriptional profiles of wild-type and cep-1(gk138) worms during normal growth and development. This analysis revealed that, indeed, a large number (331) of genes are repressed at least twofold by CEP-1 under normal growth conditions; in contrast, a much small number of genes (28) are activated by CEP-1 (Figure 1a, Table S1). Thus, in the absence of genotoxic stress CEP-1 apparently plays a major role in repressing gene expression in both the germline and soma, which is consistent with our previous findings showing that CEP-1 regulates chromosome stability in the germline and is required for resistance to starvation and hypoxia in the soma. $^{13}$

Activation of germline apoptosis and cell division arrest are triggered by UV radiation. Because the germline is abundant, representing over $50 \%$ of the nuclei in an adult, whole animal extracts can therefore be effectively used to identify changes in gene expression in the germline in response to DNA damage. Earlier reports from our group and others suggested that CEP-1 is not required for arresting germ cell proliferation in response to ionizing radiation, but these studies were performed using an unusual dominantnegative mutant, cep-1(w40), and on worms in which cep-1 function was attenuated by RNAi or by cosuppression. ${ }^{13,14}$ The availability of a strong loss-of-function allele of cep-1, gk138, allowed us to re-examine the role of CEP-1 in germ cell proliferation. In contrast to the earlier reports, in which cep-1 was shown to have no role in modulating proliferation arrest in response to ionizing radiation, we found that the cep-1(gk138) allele is defective in arresting germ cell proliferation in response to UV radiation (Figure $2 \mathrm{a}$ ). We

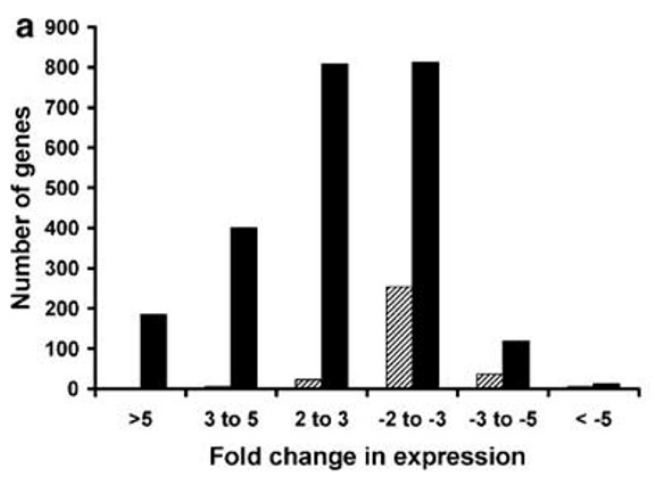

b

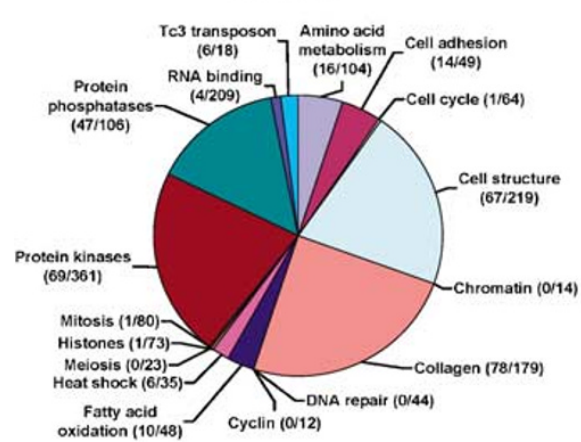

Repressed

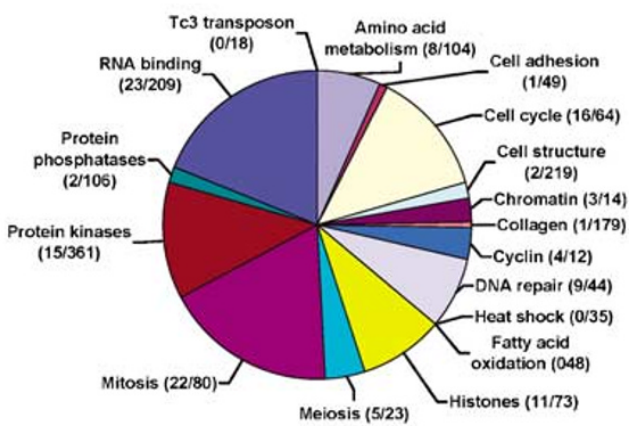

Figure 1 Effect of $100 \mathrm{Jm}^{-2}$ UV radiation on global gene expression levels. (a) Number of genes activated by UV (solid bars) compared with genes regulated by CEP-1 in the absence of UV (hatched bars). (b) Classes of genes activated (left) and repressed (right) by CEP-1 in response to UV. Gene classes were assigned using the Topomap Gene Lists program available online at http://workhorse.stanford.edu/cgi-bin/g//genelist.cgi?set $=2$ 

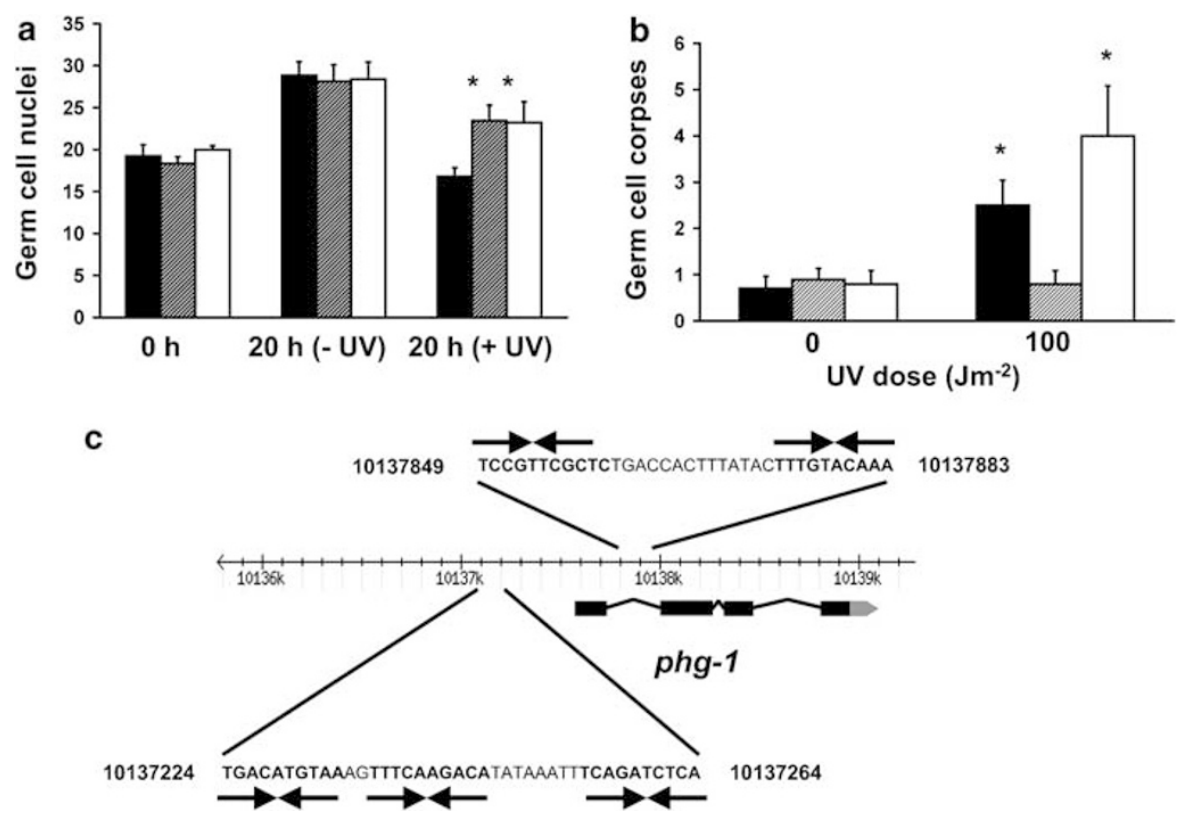

Figure 2 phg-1 and cep-1 are required to arrest germ cell proliferation in response to UV. (a) Quantification of mitotic germ cell nuclei in wild-type (solid bars), cep-1(gk138) (gray bars), and wild-type animals + phg-1(RNAi) (white bars) in the absence versus presence of $100 \mathrm{Jm}^{-2} \mathrm{UV}$ radiation. Error bars $=$ S.E.M., ${ }^{*} P<0.01$. (b) Quantification of apoptosis in pachytene region of gonads in absence versus presence of UV. Error bars $=$ S.E.M., ${ }^{\star} P<0.01$. (c) Genomic organization of, and location of p53 consensus binding sites in, the phg-1 gene. Conserved p53 consensus binding sites are present upstream of the predicted start codon and in the first intron. Arrows depict the head-to-tail arrangements of the half-sites in the p53-binding sites

also found that UV, similar to ionizing radiation, activates CEP-1-dependent apoptosis in the germline (Figure 2b). Given this strong CEP-1-dependent checkpoint response to UV, we sought to characterize the genes regulated by CEP-1 in response to this stress.

\section{A large number of genes are activated and repressed by CEP-1 in response to UV radiation. To identify the set of C. elegans genes that are regulated by CEP-1 in response to UV radiation, we compared genome-wide expression profiles of wild-type animals and cep-1(gk138) mutants. We isolated RNA $4 \mathrm{~h}$ after treatment with UV to identify early responsive genes and to minimize enriching the data set with secondary targets (Materials and Methods). In response to UV irradiation, we observed a 50-fold increase in the number of CEP-1-activated genes (from 28 to 1394) and a 2.8-fold increase in the number of CEP-1-repressed genes (from 331 to 942) (Figure 1a, Table S1). Both activated and repressed genes represent a wide range of biological functions (Figure 1b) and are generally evenly distributed across the genome; however, the $\mathrm{X}$ chromosome contains substantially fewer repressed genes compared with those on the autosomes (Table S1). We found that $77 \%$ of the genes repressed by CEP-1 plus UV are expressed in the germline ${ }^{21}$ consistent with the pivotal role CEP-1 plays in mediating the germline checkpoint response.}

Coregulative clustering of CEP-1-regulated genes. We compared our results with data summarized from numerous microarray experiments carried out under a variety of conditions and genetic backgrounds in $C$. elegans and found that UV dramatically alters the distribution of CEP-1- regulated genes across coregulated clusters. ${ }^{22}$ In the absence of UV, 12 out of 28 genes upregulated by CEP-1 cluster into functional classes that are also enriched in the germline (Table 1). Although UV radiation caused a dramatic increase in the number of germline-enriched genes (from 12 to 160), the majority of genes upregulated by CEP-1 plus UV were not normally enriched in the germline (Table 1). In contrast, $41 \%$ of genes repressed by CEP- 1 in the absence of UV were germline enriched, which increased to $77 \%$ germline enriched in the presence of UV (Table 2). We also found that an overwhelming number of genes repressed by CEP-1 under normal growth conditions were also activated by CEP-1 in response to UV (307 out of 331 genes), suggesting that UV may cause CEP-1 to toggle its regulatory output from repression to activation for certain genes involved in the UV response.

p53 consensus binding sites in CEP-1-regulated genes. In an effort to identify putative direct targets of CEP-1, we analyzed CEP-1-activated genes for p53 consensus DNA-binding sites. ${ }^{23}$ Vertebrate p53 binds two copies of the palindromic DNA consensus sequence RRRCWWGYYY, and CEP-1 binds an oligonucleotide containing the human p53 consensus motif in vivo and in vitro. ${ }^{14,15}$ We searched the $C$. elegans genome for pairs of p53 consensus binding sites and found that $\sim 25 \%$ of CEP-1activated genes contain potential p53-binding sites in their promoters and introns (Table S2). These sites were nonrandomly distributed across the genome: although approximately half of the genes on chromosomes I and II contain these sites, there are substantially fewer in those genes on chromosomes III, IV, V and X. In many cases, we 
Table 1 Distribution of CEP-1-activated genes in specific clusters (mountains)

\begin{tabular}{|c|c|c|c|c|c|}
\hline \multirow[b]{2}{*}{$\begin{array}{l}\text { Gene cluster } \\
\text { (mountain) }\end{array}$} & \multicolumn{2}{|c|}{-UV } & \multicolumn{2}{|c|}{ +UV } & \multirow[b]{2}{*}{$\begin{array}{l}\text { Gene } \\
\text { function }\end{array}$} \\
\hline & $\begin{array}{l}\text { Identified/total } \\
\text { in mountain }\end{array}$ & $\begin{array}{c}\text { Representation } \\
\text { factor }^{a}\end{array}$ & $\begin{array}{l}\text { Identified/total } \\
\text { in mountain }\end{array}$ & $\begin{array}{c}\text { Representation } \\
\text { factor }\end{array}$ & \\
\hline 4 & & & $514 / 1195$ & 5.4 & Sperm, kinases, phosphatases \\
\hline 7 & $5 / 810$ & 3.9 & & & Germline, oocyte, meiosis, mitosis, DNA repair \\
\hline 12 & $7 / 462$ & 9.5 & & & \\
\hline 14 & & & $111 / 353$ & 4.0 & Collagen \\
\hline 16 & & & $96 / 230$ & 5.3 & Muscle, collagen \\
\hline 21 & & & $24 / 154$ & 2.0 & Lipid metabolism \\
\hline 27 & & & $13 / 87$ & 1.9 & Amino-acid metabolism, energy \\
\hline 29 & & & $18 / 40$ & 5.7 & \\
\hline 35 & & & $11 / 15$ & 9.2 & Collagen \\
\hline 36 & & & $4 / 10$ & 5.0 & Heat shock \\
\hline 37 & & & $6 / 11$ & 6.9 & Transposase \\
\hline
\end{tabular}

Genes activated twofold of greater were submitted to the Topomap gene lists (http://workhorse.stanford.edu/cgi bin/gl/gene_list.cgi?set $=2$ ) at Stanford University and compared with the database of over 500 different microarray experiments. ${ }^{2}$ Representation factor is defined as the number of overlapping genes divided by the expected number of overlapping genes drawn from two independent groups. ${ }^{22}$ Only the mountains with representation factors of 1.9 or greater are indicated in Tables 1 and 2

Table 2 Distribution of CEP-1-repressed genes that are enriched in specific clusters (mountains)

\begin{tabular}{|c|c|c|c|c|c|}
\hline \multirow[b]{2}{*}{$\begin{array}{l}\text { Gene cluster } \\
\text { (mountain) }\end{array}$} & \multicolumn{2}{|c|}{-UV } & \multicolumn{2}{|c|}{ +UV } & \multirow[b]{2}{*}{$\begin{array}{l}\text { Gene } \\
\text { function }\end{array}$} \\
\hline & $\begin{array}{l}\text { Identified/total } \\
\text { in mountain }\end{array}$ & $\begin{array}{l}\text { Representation } \\
\text { factor }\end{array}$ & $\begin{array}{l}\text { Identified/total } \\
\text { in mountain }\end{array}$ & $\begin{array}{l}\text { Representation } \\
\text { factor }\end{array}$ & \\
\hline 2 & & & $155 / 1465$ & 2.0 & Germline, oocyte \\
\hline 4 & $134 / 1195$ & 6.0 & & & Sperm, kinases, phosphatases \\
\hline 7 & & & $317 / 810$ & 7.3 & Germline, oocyte, meiosis, mitosis, DNA repair \\
\hline 8 & $29 / 803$ & 1.9 & & & Intestine, proteases \\
\hline 11 & & & $142 / 587$ & 4.5 & Germline, oocyte, meiosis, mitosis, histone, $\mathrm{Rb}$ \\
\hline 12 & & & $49 / 462$ & 2.0 & \\
\hline 15 & $16 / 247$ & 3.5 & & & \\
\hline 16 & $17 / 230$ & 4.0 & & & Muscle, collagen \\
\hline 18 & & & $21 / 190$ & 2.1 & Germline, oocyte, biosynthesis, protein synthesis \\
\hline 21 & $10 / 154$ & 3.5 & & & \\
\hline 22 & & & $29 / 151$ & 3.6 & Collagen \\
\hline 27 & $3 / 87$ & 1.9 & & & \\
\hline 36 & $4 / 10$ & 21.5 & & & Heat shock \\
\hline 37 & $1 / 11$ & 4.9 & & & \\
\hline 39 & & & $1 / 8$ & 2.3 & \\
\hline 41 & & & $1 / 7$ & 2.7 & Translation \\
\hline
\end{tabular}

Data are presented as in Table 1

found multiple CEP-1-activated genes with nearby p53 consensus sites clustered in the genome. Therefore, CEP1 orchestrates the expression of numerous UV-responsive genes, many of which appear to be organized into coordinately regulated gene clusters.

Functional requirement of CEP-1-regulated genes. Most of the annotated genes in $C$. elegans have been assayed for reduction-of-function phenotypes using $\mathrm{RNAi}^{24,25}$ and a survey of all RNAi experiments reported on Wormbase (www.wormbase.org) indicates that $13.8 \%$ of genes assayed cause embryonic lethality or sterility and $7.3 \%$ cause postembryonic phenotypes (Figure 3). Of the 321 genes that are repressed by CEP-1 under normal growth conditions, $91 \%$ have no reported RNAi phenotype. This is consistent with the observation that cep-1 knockout mutants are phenotypically grossly normal. Although five of the 28 genes (18\%) upregulated by CEP-1 under normal growth conditions

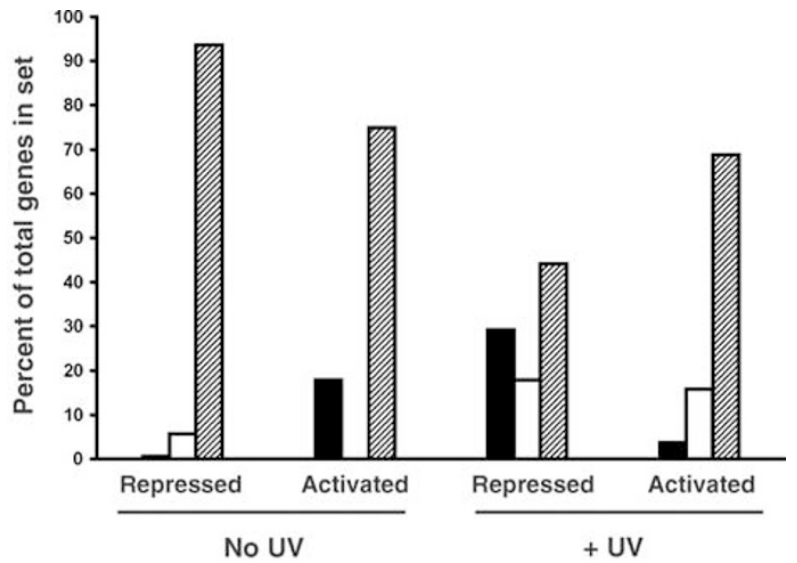

Figure 3 RNAi phenotypes of genes activated and repressed by CEP-1 in the absence and presence of UV. The fraction of genes in each data set that displays embryonic lethality and sterility (solid bars), postembryonic phenotypes (open bars) or no detectable phenotype (hatched bars) was determined by searching RNAi phenotypes using Wormbase (www.wormbase.org) 
show embryonic lethality by RNAi, no phenotype is reported for the remainder (Figure 3 ). Although only $2.4 \%$ of the genes activated by CEP-1 in response to UV exhibit RNAi-induced sterility and embryonic lethality, $21.3 \%$ of genes repressed by CEP-1 in response to UV show these phenotypes (Figure 3). The fraction of UV-induced genes that show post-embryonic RNAi phenotypes other than sterility is similar in both the CEP-1-activated and -repressed groups, but is higher compared with the fraction observed for the entire genome (Figure 3).

As most post-embryonic phenotypes were equally represented in both the UV-activated and -repressed data sets, we found twice as many genes that cause a slow growth (Gro) phenotype by RNAi among the set repressed by CEP-1 in response to UV (data not shown). Under normal growth conditions, $3.1 \%$ of repressed genes and $7.1 \%$ of CEP-1activated genes are reported to cause Gro phenotypes by RNAi (data not shown). This observation raises the possibility that an important function of CEP-1 may be to attenuate the rate of development (see below).

Coincidence of CEP-1-regulated genes and human p53-regulated relatives. Our comprehensive analysis of p53-regulated genes in $C$. elegans made it possible to compare the transcriptional profiles of genes regulated by p53 family members in human cells. Worm genes that are up- or down-regulated by CEP-1 were compared with their human orthologues that are activated or repressed by $\mathrm{p53}$, p63, and p73. $5,6,10$ This comparison revealed a substantial overlap between the human and worm gene sets (Table S3). No orthologues were found to be upregulated by CEP-1 and all three p53 family members; however, two genes encoding a tyrosine kinase (B0302.1) and caveolin (T13F2.8) were upregulated by both p53 in human cells and CEP-1 in worms. In contrast, we observed considerable overlap between CEP-1 and TAp63 $\gamma$-activated genes (Table S3): of the 128 genes induced by TAp63 $\gamma^{5}$ in human cells, Ensembl reports 25 worm orthologues, of which nearly half (10 genes) are upregulated at least 1.5 -fold by CEP-1+UV (Table S3). These findings suggest that CEP-1 may be more similar to p63 with respect to its transcription-activating properties.

In contrast, we observed considerable overlap among genes repressed by CEP-1 and human p53. In fact, more than half of the identified orthologues were found to be repressed in both humans and worms by p53 and CEP-1, respectively. We examined p53-repressed genes reported in three separate studies and compared these with their worm orthologues (Table S4). In total, of the p53-repressed genes reported in three different studies, we found that 20 out of 33 C. elegans orthologues (61\%) were also repressed by CEP-1, including a number of genes that regulate the cell cycle and DNA replication. Unfortunately, the published microarray studies for p63 and p73 focused only on activated genes. ${ }^{5,6}$ Nonetheless, these findings indicate a high degree of similarity between the p53 regulatory networks in humans and worms.

Control of germ cell proliferation by CEP-1 and PHG1. The results described above revealed that CEP-1 regulates cell proliferation in the germline in response to UV radiation. In contrast to the action of human p53, however, this response does not appear to be mediated through p21-type cyclin-dependent kinase inhibitors, as the two p21 homologues in $C$. elegans, cki-1 and -2, were not activated by UV in our experiments (Table S1). We searched the microarray data for CEP-1-activated cell cycle inhibitors and identified phg-1 (pharynx associated Gas 1 ), ${ }^{26}$ which was activated threefold by CEP-1 in response to UV. The phg-1 gene contains upstream and intronic p53-binding sites (Figure 2c, Table S2), and the human homologue Gas1 (Growth arrest-specific 1) regulates p53-dependent cell cycle arrest in serum-starved NIH 3T3 cells by a mechanism that is apparently independent of p53's ability to regulate gene expression. ${ }^{27}$ To assess its role in regulating germ cell division, we inhibited phg-1 by RNAi and quantified mitotic germ cell proliferation. We found that phg-1 is required for the arrest of germ cell proliferation in response to UV to approximately the same degree as cep-1 (Figure 2a). Further, the phenotype of cep-1(gk138); phg-1(RNAi) double mutants is not discernibly different from that of either single mutant, implying that cep-1 and phg-1 function in the same pathway (not shown). In contrast, although the cep-1(gk138) mutation effectively blocks UV-induced germ cell apoptosis, inhibiting phg-1 by RNAi does not suppress

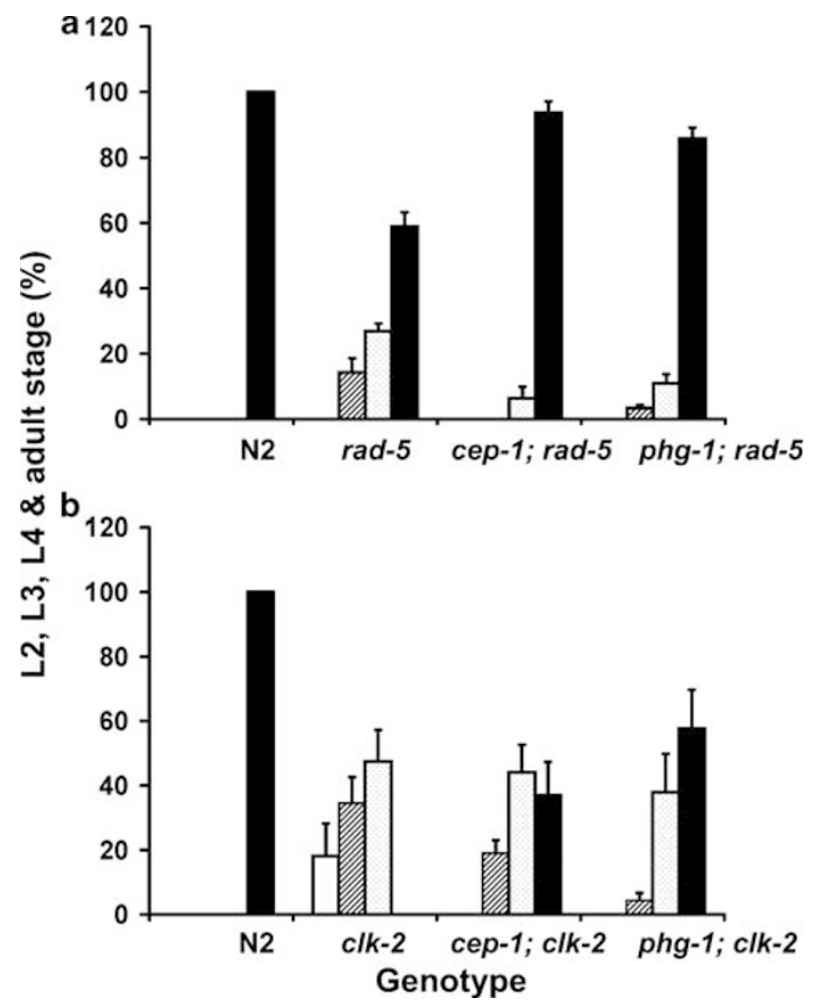

Figure 4 cep-1(-) and phg-1(RNAi) partially suppress developmental delay of two clk-2 alleles. (a) Quantification of developmental stages of wild-type (N2), $c l k-2(m n 159)$, cep-1 (gk138); clk-2(mn159), and clk-2(mn159) + phg-1(RNAi) animals after 5 days of growth from embryo stage at $15^{\circ} \mathrm{C}$. (b) Quantification of developmental stages of N2, clk-2(qm37), cep-1(gk138); clk-2(qm37), and clk2 (qm37) + phg-1(RNAi) animals after 5 days of growth from embryo stage at $15^{\circ} \mathrm{C}$. Larval stage 2 (L2) (open bars), L3 stage (hatched gray bars), L4 stage (light gray bars), and adult stage (solid bars). Error bars $=$ S.E.M. 

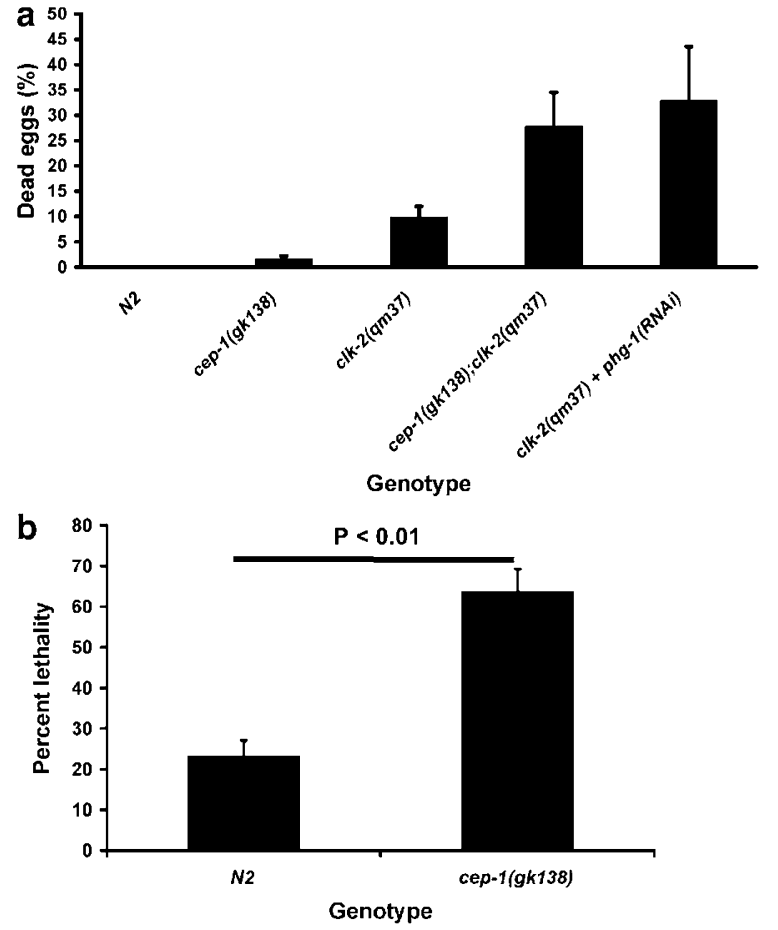

Figure 5 Rescue of developmental delay in clk-2 mutants by cep-1(-) and phg1(-) mutations is accompanied by synthetic lethality. (a) Young adult worms of the indicated genotype were allowed to lay eggs for $4 \mathrm{~h}$ and P0 adults were then removed. Embryos were counted immediately after adults were removed and unhatched eggs counted after 2 days of cultivation at $15^{\circ} \mathrm{C}$. Error bars $=$ S.E.M. ${ }^{*} P<10^{-6}$ (t-test). (b) cep-1(gk138) mutants are hypersensitive to hydroxyurea. L4 stage hermaphrodites were placed on NGM plates that contained $10 \mathrm{mM} \mathrm{HU}$ and allowed to lay eggs for $6 \mathrm{~h}$ at $20^{\circ} \mathrm{C}$. Adults were then removed and inviable F1 embryos quantified after $48 \mathrm{~h}$

apoptosis (Figure 2b). Collectively, these findings suggest that the germline cell cycle inhibitory effect of CEP-1 in response to genotoxic stress is mediated by phg-1.

Developmental rate is regulated by PHG-1 and CEP1. Among the genes known to be required for DNA damageinduced checkpoint control in the germline is $c / k-2$, originally named rad-5 based on the radiation-sensitive phenotype of rad-5 mutants. ${ }^{28}$ The allelic mutations rad-5(mn159) and clk-2(qm37) both display temperature-sensitive lethality, hypersensitivity to DNA-damaging agents, and failure to activate germline apoptosis or proliferation arrest in response to DNA damage. ${ }^{16,18}$ As both cep-1 and phg-1 perform similar roles in regulating germ cell proliferation (Figure $2 a$ ), we sought to determine whether they might also modulate the rate of development. We analyzed the rates of development of cep-1(gk138); clk-2(mn159) and cep1(gk138); clk-2(qm7) double mutants from embryogenesis to adulthood and found that the slow developmental rate conferred by both clk-2 alleles is suppressed by the cep1(gk138) mutation (Figure 4). This finding reveals that CEP-1 modulates developmental rate in the soma in the absence of CLK-2 function. Further, we found that inhibiting expression of phg- 1 by RNAi in the clk-2 mutants phenocopies the effect of $c e p-1$ (gk138), indicating that cep-1 and phg-1 likely control somatic development through the same pathway (Figure 4).
The action of CEP-1 and PHG-1 in reducing the rate of development is likely important for the viability of the animal, as restoration of developmental rates in cep-1 and phg-1 mutants results in lethality: although partially restoring normal developmental rate to clk-2 mutants, the cep1(gk138) mutation and phg-1(RNAi) also caused a substantial increase in embryonic lethality in combination with the clk-2 mutation (Figure 5a). The dying embryos arrest late in development, undergo differentiation, and do not show elevated apoptosis (not shown). Thus, cep-1 and phg-1 may be required in embryos to temporarily arrest or slow development, perhaps allowing repair of endogenous DNA damage that arises during development in clk-2 mutants.

Because clk-2 mutants are hypersensitive to replication inhibitors, ${ }^{18}$ we asked whether cep-1 might function in replication stress by treating cep-1(gk138) mutants with $10 \mathrm{mM}$ hydroxyurea (HU) and quantifying embryonic lethality in the F1 progeny. We found that cep-1 (gk138) mutants are $\sim 3$-fold more sensitive to $\mathrm{HU}$ than wild-type controls (Figure 5b), revealing that CEP-1 is required for survival during embryogenesis when replication is slowed. Collectively, these findings suggest that p53-mediated developmental checkpoints exist in the soma of $C$. elegans to coordinate replication with development and ensure that development proceeds at the proper rate.

\section{Discussion}

p53-mediated gene regulation during normal growth. Although much attention has been paid to p53 action in response to cellular stress, the function of p53 during normal growth and development is not well understood. A mutation that eliminates all p53-related activity in an animal has allowed us to identify the comprehensive set of genes regulated by p53 during normal growth.

It is noteworthy that a large number of genes are regulated by CEP-1 in the absence of stress (28 activated and 331 repressed by at least twofold). Among the activated genes are two members of the DNA replication factor $C$ family and a TATA binding protein (Table S1). This suggests that CEP-1 may be important in general transcription and replication control, consistent with our finding that cep-1 mutants are hypersensitive to the replication inhibitor HU (Figure 5b). In the absence of stress, CEP-1 functions predominantly to repress gene expression and its repressed targets are coregulated, implying that CEP-1 coordinates cellular activities during normal growth. Most (93\%) of these repressed genes are activated $>2$-fold by CEP-1 in response to UV, suggesting that genotoxicity causes CEP-1 to switch from repression to activation of many genes. $C$. elegans normally lives in the soil, where it encounters many environmental stressors; thus, CEP-1 may have evolved to modulate expression of a selected set of genes in the absence of stress to respond rapidly to environmental changes that threaten genome stability.

Range of functions regulated by p53 in response to genotoxic stress. UV caused a 50 -fold increase in the 
number of CEP-1-activated genes (Figure 1), encompassing a wide range of protein functions, as seen for vertebrate p53 family members. ${ }^{5,6,9,12}$ However, there were distinct differences between the genes regulated by CEP-1 and the human p53 family. Among the most prominent classes of CEP-1-regulated genes were collagen genes: over $40 \%$ of the $178 C$. elegans collagen genes were activated $>2$-fold in response to UV (Figure 1b). This is not likely an artifact of differences in developmental stages between the two genotypes, as no collagen genes were upregulated by CEP-1 in the absence of UV; moreover, nearly half of these genes contain strong p53 consensus binding sites (Table S2). Upregulation of collagens by CEP-1 might enhance the protective barrier of the cuticle against environmental stresses. It is interesting to note that several collagen genes have also been identified as mammalian p53 and p73 targets. ${ }^{5,6,29}$

Several stress-response proteins are regulated by CEP-1. For example, an hsp70 gene (F44E5.5) containing a p53 consensus site is UV-activated $>10$-fold by CEP-1 (Tables S2 and S3). Hsp70 is regulated by mammalian p53, indicating that these stress-response proteins are likely important effectors of the DNA damage response. ${ }^{29}$ Expression of several heat-shock genes are downregulated with age in $C$. elegans $^{30}$ and increased p53 activity has been associated with an increased rate of aging in flies and mice. ${ }^{31,32}$ It will be of interest to learn how CEP-1 activity changes with the age of the worm.

Mammalian p53 regulates DNA repair and Drosophila p53 regulates DNA repair genes in response to ionizing radiation. ${ }^{1,31}$ We were surprised to find a number of DNA repair genes (nine out of 44) that were repressed by CEP-1 in response to UV and none that were activated (Figure $1 \mathrm{~b}$, Table S1); thus, it is conceivable that CEP-1 does not regulate DNA repair in worms. A substantial number of cell cycle genes (16 out of 64) were present among those repressed by CEP-1 in response to UV. The human counterparts of at least seven of these genes (e.g., cyclins $B$ and $E$ ) have also been reported to be repressed by $\mathrm{p} 53 ;^{10,29}$ thus repression of positive cell cycle regulators in response to genotoxic stress is a conserved function of CEP-1 and p53. We found many other examples of genes that are regulated similarly by vertebrate p53 family members and CEP-1 (Tables S3 and S4), providing compelling support that the p53 transcriptional network is ancient and was retained in modern nematodes and humans.

CEP-1 regulates germline proliferation by activating phg-1. Earlier studies reported that p53 relatives, unlike their mammalian counterparts, are not required for the radiation-induced proliferation arrest that occurs in $C$. elegans and Drosophila. ${ }^{13,14,33,34}$ However, using a stronger allele of cep-1 and UV, we found that CEP-1 does indeed inhibit germline proliferation (Figure 2a). Although the p21 cyclin-dependent kinase inhibitor is a key mediator of p53-mediated cell cycle arrest in mammals, expression of the p21-like cki-1 and cki-2 genes in C. elegans does not change in response to UV. In contrast, we found that phg-1, which contains p53 consensus sites (Figure 2c) and is activated $\sim$ 3-fold by CEP-1 (Table S1), is required for germline arrest

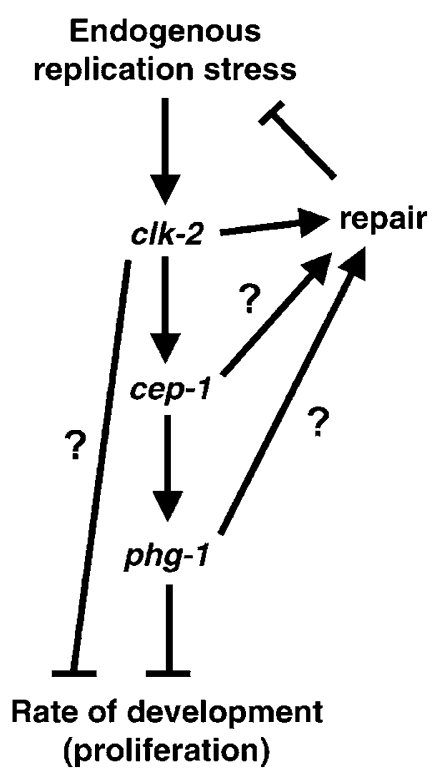

Figure 6 Model for control of developmental timing by CEP-1 and PHG-1. clk-2 mutants are defective in repairing replication errors occurring during development. The accumulation of replication errors activates cep-1 to slow development and cellular proliferation via phg-1 to permit repair. Because the suppression of the growth delay is partial, there are likely other genes downstream of $c l k-2$ that function independent of cep-1 and phg-1 to slow growth

(Figure 2a), but not UV radiation-induced germ cell apoptosis. Thus, we propose that phg-1 lies downstream of cep-1 in a branch of the DNA damage pathway that controls germ cell proliferation but not apoptosis (Figure 6). PHG-1 is the worm equivalent of human Gas1, which cooperates with p53 to block proliferation of mammalian cells. ${ }^{26,27,35}$ Gas1 has been proposed to regulate cell division through physical association with, rather than transcriptional activation by, p53. However, the presence of p53 consensus sites in the Gas1 promoter $^{36}$ raises the possibility that it is also transcriptionally regulated by p53 in humans.

Regulation of developmental rate by CEP-1 and PHG-1. The clk-2 gene controls longevity and the rate of developmental progression, ${ }^{20}$ and is required to transmit DNA damage signals to CEP-1 in the germline. ${ }^{18}$ We found that the reduced growth rate $c / k-2$ mutants is suppressed by both cep-1(gk138) and phg-1(RNAi). Although clk-2 is required for the $S$ phase replication checkpoint, we propose that slower growth rates might result from unrepaired replication errors arising during development in these mutants.

Suppression of the developmental delay in clk-2 mutants by cep-1 or phg-1 mutations was accompanied by synthetic embryonic lethality (Figure 5a), suggesting that CEP-1 and PHG-1 may participate in the repair process or modulate the cell cycle in a way that allows sufficient time for repair of replication errors. Thus, we suggest that CEP-1 monitors replication errors during development and maintains a normal developmental progression rate by regulating PHG-1 (Figure 6). 


\section{Materials and Methods}

Strains and RNAi. All strains were grown at 15 or $20^{\circ} \mathrm{C}$ as described previously. ${ }^{37}$ The wild-type strain used was C. elegans variety Bristol (N2). Alleles used in this study were: linkage group I, cep-1(gk138); linkage group III, $\left.c / k-2(m n 159),{ }^{28} c k-2(q m 37)\right)^{19}$

RNA interference was carried out by the feeding method. ${ }^{24,38}$ Briefly, bacterial strains expressing dsRNA corresponding to the phg-1 gene were grown overnight in $\mathrm{LB}+100 \mu \mathrm{g} / \mathrm{ml}$ ampicillin and $500 \mu \mathrm{g} / \mathrm{ml}$ tetracycline. Overnight cultures were plated on NGM plates supplemented with $2.5 \mathrm{mM} \mathrm{IPTG}$ and allowed to grow $24 \mathrm{~h}$ at room temperature. Worms were then placed on the RNAi feeder plates and grown for one generation. The F1 progeny at the L3 stage were then transferred to fresh RNAi feeder plates and synchronized for cell cycle and apoptosis analysis. For lethality and developmental timing experiments, several young adult PO hermaphrodites were placed on fresh OP50 or RNAi feeder plates and allowed to deposit eggs for $8-12 \mathrm{~h}$ at 15 or $20^{\circ} \mathrm{C}$. PO's were then removed from the plates, the number of eggs counted, and their developmental stage assessed using a dissecting microscope each day. Unhatched eggs were counted $24 \mathrm{~h}$ after P0's were removed and total adults were counted $72 \mathrm{~h}$ later. Lethality and rates of development were compared with those of N2 animals raised on phg-1(RNAi) feeder plates as well as on the OP50 control bacteria. We observed no effects of phg-1(RNAi) on growth rates or lethality of N2 worms (data not shown).

To assess hydroxyurea sensitivity, we prepared NGM plates with $10 \mathrm{mM}$ hydroxyurea in the media as described by Ahmed et al. ${ }^{18} \mathrm{P} 0$ animals at the $L 4$ stage were placed on plates and allowed to lay eggs for $6 h$; they were then removed and the deposited eggs counted. Unhatched eggs were counted after $48 \mathrm{~h}$ and percent embryonic lethality was determined in triplicate.

Microarrays. Microarrays were constructed as described previously. ${ }^{21,39,40}$ The set of 19213 primer pairs corresponding to 94\% of the genes in the genome was used to PCR-amplify gene fragments as described. ${ }^{40}$ Of these, 18010 produced a single band of the correct size. Reverse transcription, labeling, and hybridization to the arrays were carried out as described elsewhere. ${ }^{39}$ RNA was isolated in triplicate from carefully synchronized populations of $\mathrm{N} 2$ and cep-1(gk138) young adult worms under normal conditions of growth and four hours after treatment with $250 \mathrm{Jm}^{-2} \mathrm{UV}$ radiation. Cy5-dUTP (red) was used to label cDNA from wild-type worms and Cy3dUTP (green) was used to label cDNA from cep-1 (gk138) mutants. Measurements from the three individual RNA isolations were averaged and the ratio of wild-type to cep-1 mutants was calculated. Measurements from the three individual RNA isolations were averaged and the ratio of wild type to cep-1 mutants was calculated. Comparisons were made between genotypes by subtracting the mean log value of one ratio from another, and the significance of the difference was evaluated using Student's $t$-test for two populations. We chose a combination of a twofold difference with a $t$-value exceeding $99 \%$ confidence $(P<0.01)$ to reduce the probability of obtaining false positives. We note that although the cep-1(gk138) strain was outcrossed four times to wild type, it is possible that residual background mutations may remain in this strain that could influence some of the gene expression data.

Germ cell proliferation and apoptosis. For germ cell proliferation measurements, animals were synchronously grown to the $L 4$ stage at $20^{\circ} \mathrm{C}$ and treated with UV-C radiation on NGM plates using a Stratalinker (Stratagene, Inc.). Immediately after irradiation, worms were transferred to fresh plates and germ nuclei were counted under DIC microscopy within a defined volume of the mitotic germline $20 \mathrm{~h}$ post-UV treatment. Unirradiated controls were quantified in parallel and mitotic germ nuclei of $L 4$ animals were counted and compared with the number of germ nuclei of animals $20 \mathrm{~h}$ after the $L 4$ molt. To assess the effects of UV on germ cell apoptosis, worms were allowed to develop to the young adult stage $(24 \mathrm{~h}$ after L4 stage) at $20^{\circ} \mathrm{C}$, treated with UV, and apoptotic corpses were quantified by DIC microscopy in the pachytene region of the gonad $24 \mathrm{~h}$ later. Because the germline continuously proliferates throughout adulthood, the effect of DNA-damaging agents on apoptosis is more pronounced in young adults compared with $L 4$ animals, which have smaller germlines and therefore fewer corpses after UV treatment.

Acknowledgements. The cep-1(gk138) mutant strain was provided by the C. elegans Gene Knockout Consortium, Vancouver, BC. Some of the strains were provided by the C.elegans Genetics Center, which is funded by the NIH National Center for Research Resources. Supported by funding from the Tri-Counties Blood Bank and the Cancer Center of Santa Barbara, The Hospital for Sick Children Research Institute, the Canada Foundation for Innovation and the Canadian
Institutes of Health Research (WBD), and a grant \# CA95943 from the National Institute of Health (JHR).

1. Levine AJ. p53, the cellular gatekeeper for growth and division. Cell 1997; 88: 323-331.

2. el-Deiry WS, Tokino T, Velculescu VE, Levy DB, Parsons R, Trent JM et al. WAF1, a potential mediator of p53 tumor suppression. Cell 1993; 75: 817-825.

3. Brugarolas J, Chandrasekaran C, Gordon JI, Beach D, Jacks T, Hannon GJ. Radiationinduced cell cycle arrest compromised by p21 deficiency. Nature 1995; 377: 552-557.

4. Deng C, Zhang P, Harper JW, Elledge SJ, Leder P. Mice lacking p21CIP1/WAF1 undergo normal development, but are defective in G1 checkpoint control. Cell 1995; 82: 675-684.

5. Osada M, Park HL, Nagakawa Y, Yamashita K, Fomenkov A, Kim MS et al. Differential recognition of response elements determines target gene specificity for p53 and p63. Mol Cell Biol 2005; 25: 6077-6089

6. Fontemaggi G, Kela I, Amariglio N, Rechavi G, Krishnamurthy J, Strano S et al. Identification of direct $p 73$ target genes combining DNA microarray and chromatin immunoprecipitation analyses. J Biol Chem 2002; 277: 43359-43368.

7. Flores ER, Tsai KY, Crowley D, Sengupta S, Yang A, McKeon F et al. p63 and p73 are required for p53-dependent apoptosis in response to DNA damage. Nature 2002; 416: 560-564.

8. Senoo M, Manis JP, Alt FW, McKeon F. p63 and p73 are not required for the development and p53-dependent apoptosis of T cells. Cancer Cell 2004; 6: 85-89.

9. Mirza A, Wu Q, Wang L, McClanahan T, Bishop WR, Gheyas F et al. Global transcriptional program of $\mathrm{p} 53$ target genes during the process of apoptosis and cell cycle progression. Oncogene 2003; 22: 3645-3654.

10. Kannan K, Amariglio N, Rechavi G, Jakob-Hirsch J, Kela I, Kaminski N et al. DNA microarrays identification of primary and secondary target genes regulated by p53. Oncogene 2001; 20: 2225-2234.

11. Kannan K, Kaminski N, Rechavi G, Jakob-Hirsch J, Amariglio N, Givol D. DNA microarray analysis of genes involved in p53 mediated apoptosis: activation of Apaf-1. Oncogene 2001; 20: 3449-3455.

12. Liu S, Mirza A, Wang L. Generation of p53 target database via integration of microarray and global p53 DNA-binding site analysis. Methods Mol Biol 2004; 281: 33-54.

13. Derry WB, Putzke AP, Rothman JH. Caenorhabditis elegans p53: role in apoptosis, meiosis, and stress resistance. Science 2001; 294: 591-595.

14. Schumacher B, Hofmann K, Boulton S, Gartner A. The C. elegans homolog of the p53 tumor suppressor is required for DNA damage-induced apoptosis. Curr Biol 2001; 11: 1722-1727.

15. Huyen Y, Jeffrey PD, Derry WB, Rothman JH, Pavletich NP, Stavridi ES et al. Structural Differences in the DNA Binding Domains of Human $\mathrm{p53}$ and Its C. elegans Ortholog Cep-1. Structure (Camb) 2004; 12: 1237-1243.

16. Gartner A, Milstein S, Ahmed S, Hodgkin J, Hengartner MO. A conserved checkpoint pathway mediates DNA damage-induced apoptosis and cell cycle arrest in $C$. elegans. Molecular Cell 2000; 5: 435-443.

17. Hofmann ER, Milstein S, Boulton SJ, Ye M, Hofmann JJ, Stergiou L et al. Caenorhabditis elegans HUS-1 Is a DNA damage checkpoint protein required for genome stability and EGL-1-mediated apoptosis. Curr Biol 2002; 12: 1908-1918.

18. Ahmed S, Alpi A, Hengartner MO, Gartner A. C. elegans RAD-5/CLK-2 defines a new DNA damage checkpoint protein. Curr Biol 2001; 11: 1934-1944

19. Lakowski B, Hekimi S. Determination of life-span in Caenorhabditis elegans by four clock genes. Science 1996; 272: 1010-1013.

20. Benard C, McCright B, Zhang Y, Felkai S, Lakowski B, Hekimi S. The C. elegans maternaleffect gene clk-2 is essential for embryonic development, encodes a protein homologous to yeast Tel2p and affects telomere length. Development 2001; 128: 4045-4055

21. Reinke V, Gil IS, Ward S, Kazmer K. Genome-wide germline-enriched and sex-biased expression profiles in Caenorhabditis elegans. Development 2004; 131: 311-323.

22. Kim SK, Lund J, Kiraly M, Duke K, Jiang M, Stuart JM et al. A gene expression map for Caenorhabditis elegans. Science 2001; 293: 2087-2092.

23. el-Deiry WS, Kern SE, Pietenpol JA, Kinzler KW, Vogelstein B. Definition of a consensus binding site for p53. Nat Genet 1992; 1: 45-49.

24. Fraser AG, Kamath RS, Zipperlen P, Martinez-Campos M, Sohrmann M, Ahringer J. Functional genomic analysis of $C$. elegans chromosome I by systematic RNA interference. Nature 2000; 408: 325-330

25. Kamath RS, Fraser AG, Dong Y, Poulin G, Durbin R, Gotta M et al. Systematic functional analysis of the Caenorhabditis elegans genome using RNAi. Nature 2003; 421: 231-237.

26. Agostoni E, Gobessi S, Petrini E, Monte M, Schneider C. Cloning and characterization of the C. elegans gas1 homolog: phas-1. Biochim Biophys Acta 2002; 1574: 1-9.

27. Del Sal G, Ruaro EM, Utrera R, Cole CN, Levine AJ, Schneider C. Gas1-induced growth suppression requires a transactivation-independent p53 function. Mol Cell Biol 1995; 15: 7152-7160.

28. Hartman PS, Herman RK. Radiation-sensitive mutants of Caenorhabditis elegans. Genetics 1982; 102: 159-178.

29. Zhao R, Gish K, Murphy M, Yin Y, Notterman D, Hoffman WH et al. Analysis of p53regulated gene expression patterns using oligonucleotide arrays. Genes Dev 2000; 14: 981-993.

30. Lund J, Tedesco P, Duke K, Wang J, Kim SK, Johnson TE. Transcriptional profile of aging in C. elegans. Curr Biol 2002; 12: 1566-1573. 
31. Brodsky MH, Weinert BT, Tsang G, Rong YS, McGinnis NM, Golic KG et al. Drosophila melanogaster MNK/Chk2 and p53 regulate multiple DNA repair and apoptotic pathways following DNA damage. Mol Cell Biol 2004; 24: 1219-1231.

32. Tyner SD, Venkatachalam S, Choi J, Jones S, Ghebranious $\mathrm{N}$, Igelmann $\mathrm{H}$ et al. p53 mutant mice that display early ageing-associated phenotypes. Nature 2002; 415: 45-53.

33. Ollmann M, Young LM, Di Como CJ, Karim F, Belvin M, Robertson S et al. Drosophila p53 is a structural and functional homolog of the tumor suppressor p53. Cell 2000; 101: 91-101.

34. Brodsky MH, Nordstrom W, Tsang G, Kwan E, Rubin GM, Abrams JM. Drosophila p53 binds a damage response element at the reaper locus. Cell 2000; 101: 103-113.

35. Ruaro EM, Collavin L, Del Sal G, Haffner R, Oren M, Levine AJ et al. A proline-rich motif in p53 is required for transactivation-independent growth arrest as induced by Gas1. Proc Natl Acad Sci USA 1997; 94: 4675-4680.
36. Hoh J, Jin S, Parrado T, Edington J, Levine AJ, Ott J. The p53MH algorithm and its application in detecting p53-responsive genes. Proc Natl Acad Sci USA 2002; 99: 84678472.

37. Brenner S. The genetics of Caenorhabditis elegans. Genetics 1974; 77: 71-94.

38. Kamath RS, Martinez-Campos M, Zipperlen P, Fraser AG, Ahringer J. Effectiveness of specific RNA-mediated interference through ingested double-stranded RNA in Caenorhabditis elegans. Genome Biol 2 2001; RESEARCH0002.

39. Reinke V, Smith HE, Nance J, Wang J, Van Doren C, Begley R et al. A global profile of germline gene expression in C. elegans. Molecular Cell 2000; 6: 605-616.

40. Jiang M, Ryu J, Kiraly M, Duke K, Reinke V, Kim SK. Genome-wide analysis of developmental and sex-regulated gene expression profiles in Caenorhabditis elegans Proc Natl Acad Sci USA 2001; 98: 218-223.

Supplementary Information accompanies the paper on Cell Death and Differentiation website (http://www.nature.com/cdd) 